\title{
473575 - COMPATIBILITY OF RINGER'S LACTATE WITH AS-3 PACKED RED BLOOD CELLS
}

\author{
Khala Albert, BSc, Joel Parlow, MD FRCPC, Janet vanVlymen, MD FRCPC, Paula \\ James, MD FRCPC \\ Anesthesiology, Queen's University, Kingston General Hospital, Kingston, ON, \\ Canada
}

Introduction: Ringer's lactate (RL) is a physiologic solution that has advantages over normal saline (NS) for resuscitation of hypovolemia. The current standards set by CBS and the AABB state that Ringer's lactate (RL) should not be transfused wtih packed red blood cells (PRBC) because of a theoretical risk of clotting due to calcium in RL binding the citrate in the blood preservative(1). In past studies with CPDA preserved PRBC, RL was shown to cause clotting at very high dilutions. To date there have been no reports about the safety of mixing RL with PRBC stored with the currently used preservative, AS-3. The purpose of this study was to test whether RL, as compared with NS, leads to a risk of clotting when combined with AS-3 PRBC in a clinically relevant scenario. Methods: Following ethics approval, 8 unused units of AS-3 PRBC were obtained for this study. Two parallel studies were performed at a time, comparing RL with NS as the diluent. In each study, a unit of PRBC was diluted with a crystalloid volume of $150 \mathrm{~mL}$ and infused using gravity flow through standard blood filter tubing. A series of progressively more dilute samples of $20 \mathrm{~mL}$ were collected after adding $75,100,125$, 150, 175 and 200mL of crystalloid to the bag of PRBC. Five $\mathrm{mL}$ of each sample were pushed through a 40 micron filter. All of the filters were visually examined for the presence of clots. In the remaining $15 \mathrm{~mL}$ thrombin generation was measured using ELISA to quantify the amount of F1+2 (the breakdown products of thrombin generation). The whole procedure was repeated a total of 4 times.

Results: No filters in any of the NS and RL mixtures contained evidence of clot or debris, and all allowed free passage of the blood-crystalloid mixtures. In the NS group, the F1+2 levels ranged from 2.7 to $38.0 \mathrm{pmol} / \mathrm{L}$. In the RL group, the F1+2 levels ranged from 3.2 to $289.7 \mathrm{pmol} / \mathrm{L}$, which was still below physiologic levels(Figure 1)(2).

Discussion: There was no visible or molecular evidence of activation of the clotting cascade in either the RL or NS groups. Based on a reference range of F1+2 of 0.32 to 1.2 $\mathrm{nmol} / \mathrm{l}(\mathrm{n}=95)$ all of the dilutions studied had less F1+2 than the normal physiologic level(2). In situations requiring large volumes of fluid and blood replacement, RL may be preferable to NS. The current study strongly supports that RL can be transfused with AS3 PRBC without evidence of clotting or activation of the coagulation cascade.

References: 1.Transfusion. 1975; 15(3):250-255 2.Throm \& Haem.1991;65(2):153-159 


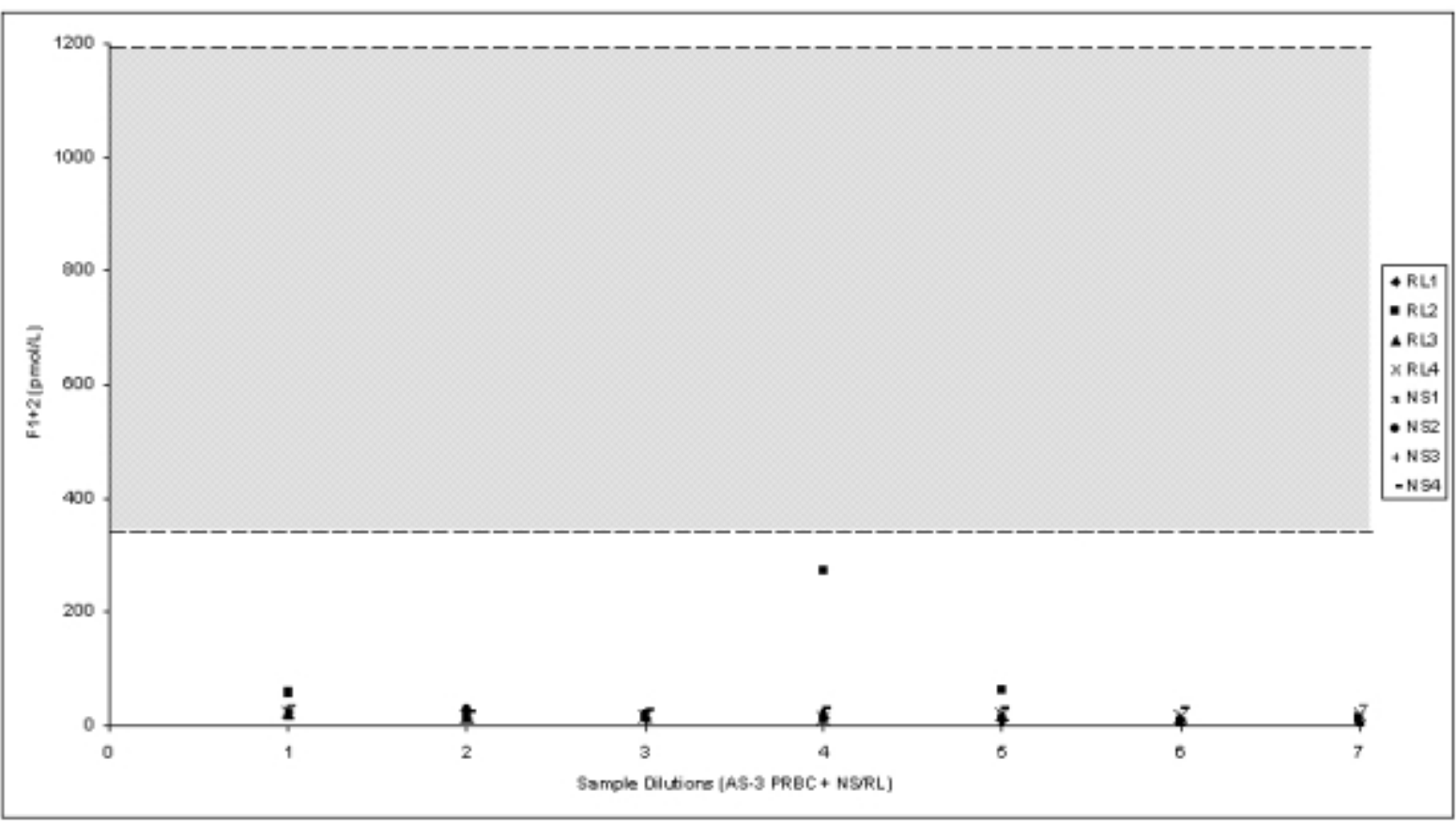

Figure 1. Mean F1+2 in duplicates of increasingly dilute mixtures of AS-3 PRBC and crystalloid (NS or RL). Area enclosed by dotted lines corresponds to physiologic F1+2 in healthy volunteers (2). 